\section{Dr. Fitzcharles, et al, reply}

To the Editor:

We appreciate the comments of Drs. Ferrari and Russell ${ }^{1}$ regarding our study that examined factors associated with disability in persons with fibromyalgia $(\mathrm{FM})^{2}$. We observed that $31 \%$ of patients with FM in our cohort of 248 patients were disabled and receiving disability payments for FM. The disabled patients with FM were more symptomatic, used more medications, and were more likely to have previously worked in physically demanding jobs. Drs. Ferrari and Russell raise the issue of symptom exaggeration by some persons with FM as a means of obtaining disability payments and suggest that effort testing and symptom exaggeration testing should be an essential component in evaluation of disability due to FM.

We agree that the extremely high rate of disability due to FM is worrisome, and that it is likely that there are some persons with FM who may be exaggerating subjective symptoms for reasons of secondary gain. We also acknowledge that the physician assessment of the severity of subjective complaint is fraught with challenges, requiring the physician to both record the complaint as presented by the patient, and also to assess the validity of the symptom report. Effort testing cannot, however, at this time be applied as a universal tool to validate symptom severity in FM, or be used as a measurement to adjudicate disability. Measurement of effort in this setting is being used as a surrogate for symptom severity, an association that has not been demonstrated to date. The critical question in the evaluation of subjective complaints as a reason for disability remains the severity of symptoms, which in turn affects functional status. A full clinical evaluation that includes assessment of day-to-day activities, information from all treating healthcare professionals, and careful observation during assessment for evidence of discrepancies in clinical findings should still remain the standard by which functionality is assessed, rather than failure on a memory effort test ${ }^{3}$.

It is also possible that some patients seeking disability status or currently identified as disabled need to maintain an illness role and thus cannot afford to get better. We agree that motivation toward health or alternatively toward disability likely contributes to symptom perception by the patient, and those who have been in a prolonged vortex of illness will lose motivation to return to health. Alternatively, returning to a work environment that is less satisfactory and enjoyable may preclude efforts toward health.
It is innate that physicians should be empathetic and trust the veracity of a patient report. We would therefore caution against a global mistrust toward patients with FM, especially because the condition to date still defies our understanding. Physicians should not compromise care of the many persons with FM as a result of the likely few who abuse the system. Rather than stating that, according to Ferrari and Russell, "FM is an illness characterized in part by a lack of face validity," we contend that suggesting that "effort testing and symptom exaggeration testing are essential in assessing patients with FM presenting for disability-related evaluations" truly lacks face validity.

MARY-ANN FITZCHARLES, MB, ChB, Alan Edwards Pain Management Unit, and Division of Rheumatology, McGill University Health Centre, Montreal; PETER A. STE-MARIE, BA, LLB, Alan Edwards Pain Management Unit, McGill University Health Centre; EMMANOUIL RAMPAKAKIS, PhD, JSS Medical Research, St-Laurent, and Jewish General Hospital, McGill University; JOHN S. SAMPALIS, PhD, JSS Medical Research, St-Laurent, and Jewish General Hospital, McGill University; YORAM SHIR, MD, Alan Edwards Pain Management Unit, McGill University Health Centre, Montreal, Quebec, Canada. Address correspondence to M.A. Fitzcharles, Montreal General Hospital, 1650 Cedar Ave., Montreal H3G 1A4, Quebec, Canada.

E-mail: mary-ann.fitzcharles@muhc.mcgill.ca

\section{REFERENCES}

1. Ferrari R, Russell AS. Effort testing and symptom exaggeration testing essential in assessing patients with fibromyalgia for disability. J Rheumatol 2016;43:2077.

2. Fitzcharles MA, Ste-Marie PA, Rampakakis E, Sampalis JS, Shir Y. Disability in fibromyalgia associates with symptom severity and occupation characteristics. J Rheumatol 2016;43:931-6.

3. Gervais RO, Russell AS, Green P, Allen LM 3rd, Ferrari R, Pieschl SD. Effort testing in patients with fibromyalgia and disability incentives. J Rheumatol 2001;28:1892-9.

J Rheumatol 2016;43:2077; doi:10.3899/jrheum.160991 